\title{
A Tunable "Ancient Coin"-Type Perfect Absorber with High Refractive Index Sensitivity and Good Angular Polarization Tolerance
}

\author{
Hao Luo ${ }^{1,+}$, Qianyi Shangguan ${ }^{2,+}$, Yinting $\mathrm{Yi}^{3}$, Shubo Cheng ${ }^{2, *}$, Yougen $\mathrm{Yi}^{3, *}$ and Zhizhong $\mathrm{Li}^{4}$ \\ 1 School of Science, Southwest University of Science and Technology, Mianyang 621010, China; \\ luoshijia224@163.com \\ 2 School of Physics and Optoelectronic Engineering, Yangtze University, Jingzhou 434023, China; \\ qianyisg@126.com \\ 3 College of Physics and Electronics, Central South University, Changsha 410083, China; sangzhen@csu.edu.cn \\ 4 Basic Teaching Department, Neusoft Institute Guangdong, Foshan 528000, China; lizhizhong114@yeah.net \\ * Correspondence: shubocheng@yangtzeu.edu.cn (S.C.); yougenyi@csu.edu.cn (Y.Y.); \\ Tel./Fax: +86-0731-88836457 (Y.Y.) \\ + These authors contribute equally to this article.
}

Citation: Luo, H.; Shangguan, Q.; Yi, Y.; Cheng, S.; Yi, Y.; Li, Z. A Tunable

"Ancient Coin"-Type Perfect

Absorber with High Refractive Index Sensitivity and Good Angular Polarization Tolerance. Coatings 2021, 11, 814. https://doi.org/10.3390/ coatings11070814

Academic Editor: Dimitrios Tasis

Received: 14 May 2021

Accepted: 29 June 2021

Published: 6 July 2021

Publisher's Note: MDPI stays neutral with regard to jurisdictional claims in published maps and institutional affiliations.

Copyright: (c) 2021 by the authors. Licensee MDPI, Basel, Switzerland. This article is an open access article distributed under the terms and conditions of the Creative Commons Attribution (CC BY) license (https:/ / creativecommons.org/licenses/by/ $4.0 /)$.

\begin{abstract}
In this paper, we design and present a graphene-based "ancient coin"-type dual-band perfect metamaterial absorber, which is composed of a silver layer, silicon dioxide layer, and a top "ancient coin" graphene layer. The absorption performance of the absorber is affected by the hollowed-out square in the center of the graphene layer and geometric parameters of the remaining nano disk. The optical properties of graphene can be changed by adjusting the voltage, to control the absorption performance of the absorber dynamically. In addition, the centrally symmetric pattern structure greatly eliminates the polarization angle dependence of our proposed absorber, and it exhibits good angular polarization tolerance. Furthermore, the proposed "ancient coin"-type absorber shows great application potential as a sensor or detector in biopharmaceutical, optical imaging, and other fields due to its strong tunability and high refractive index sensitivity.
\end{abstract}

Keywords: perfect absorber; graphene; tunable dual-band; "ancient coin"-type array

\section{Introduction}

The concept of metamaterials (MMs) was first proposed by Rodger M. Walser in 2001 to describe an artificially designed composite material with a periodic unit structure [1]. The overall characteristics of metamaterials are determined by the characteristics of the periodic structure unit cell, which has little to do with the material itself [2-4]. The constitutive parameters of metamaterials, that is, the effective permittivity $\varepsilon_{\text {eff }}$ and permeability $\mu_{\text {eff, }}$, can be used to represent the electromagnetic properties of metamaterials, and they can also be artificially adjusted to obtain certain kinds of electromagnetic properties we need [5,6]. When $\varepsilon_{\text {eff }}$ and $\mu_{\text {eff }}$ are negative, the incident light couples with the plasmon wave on the surface of the material, causing the energy of the incident light to be absorbed $[7,8]$. The peculiar electromagnetic properties of metamaterials have not been found in natural materials and traditional composite materials [9-12], for example, backward waves and negative refractive index [13-15]. Based on these excellent optical and electromagnetic properties of metamaterials, researchers have expanded many applications of metamaterials, such as perfect lenses, stealth, photovoltaic solar cells, and absorbers [16-18].

Among them, the metamaterial absorber (MMA), which is broadly used in burgeoning fields such as sensing, imaging, thermal radiation, and solar power generation, has attracted widespread attention [19-21]. The first MMA appeared in 2008, and Landy successfully demonstrated it at microwave frequencies. They achieved an absorption rate of $88 \%$ by manipulating the $\varepsilon_{\text {eff }}$ and $\mu_{\text {eff }}$ of the metamaterial independently [22]. Since then, 
many researchers have developed broadband absorption based on the narrowband singlefrequency absorber presented by Landy, and its absorption efficiency has been greatly improved [23-25]. Nowadays, various perfect absorbers with different characteristics are becoming active in increasingly more fields [26-28]. However, these absorbers mentioned above exhibit a defect: once the structure of the absorber is proposed, the optical properties cannot be changed flexibly. This is mainly because several metamaterial perfect absorbers that have been proposed are usually based on metal structures, and the physical properties of metals are relatively stable, so the absorbers are static, and the range of their working frequency bands is also fixed [29]. This defect greatly limits the possibilities for dynamic adjustment of absorber performance. Although there are some technologies that can achieve dynamic frequency adjustment, such as laser power illumination, fluid filling, and temperature change, most of these physical adjustment methods are complicated, and the efficiency of adjustment is not enough for the practical use of equipment [30-34]. Therefore, realizing a tunable perfect absorber has become a new hot spot in current research, and it is also an inevitable trend in its practical application.

The problem had not been settled until the emergence of graphene. Graphene is a hexagonal honeycomb material formed by densely packed carbon atoms on a twodimensional plane. It has outstanding optical, electrical, and mechanical properties, such as fast carrier mobility and high optical transparency [35-38]. In the far-infrared and terahertz band, a striking feature of graphene unlike traditional precious metals is that its surface plasmon resonance (SPR) has an extremely strong field confinement and lower ohmic loss. It can be closely combined with deep subwavelength nano-level light [39-45], which greatly promotes its light absorption rate in many devices, such as photoelectric sensors, optical detectors, waveguides, and ultra-fast switches [46-50]. In addition, the most significant feature of graphene plasmons is that the surface conductivity of graphene can be adjusted by changing the Fermi level or chemical potential. Thus, the application of an external gate voltage and the method of light-induced doping are approaches to convert graphene between the metallic state and the dielectric state to control the optical properties of graphene [51-53]. This means that the dynamic tunability of graphene makes it unnecessary to rebuild a new structure to change the characteristics of the absorber. The macroscopic characteristics can also be manipulated by changing the microstructure. Nowadays, many absorber structures based on graphene plasmons have been designed [54-57], but most of these absorbers use single-band absorption. Although some dual-frequency absorbers have been proposed [58], they still have some shortcomings. For example, these absorbers are always linked with polarization sensitivity, and the commonly used multi-frequency absorbers designed based on the frequency superposition method $[59,60]$ are complicated in structure. In addition, in actual manufacturing processes, a series of technical problems such as large battery size and high requirements for manufacturing technology accuracy can occur [61]. Therefore, a new method to realize dual-band perfect metamaterial absorbers based on graphene must be found urgently.

In this work, a periodic arrangement of dual-band metamaterial perfect absorbers based on graphene plasmons was designed and investigated. By stacking metal and graphene layers, the condition of impedance matching was satisfied, causing the absorption rate of light of the structure to reach approximately $100 \%$. The graphene used in the absorber made it possible to achieve dynamic frequency adjustment by applying an external gate voltage or by the method of light-induced doping, which greatly saves the cost of fabrication. In addition, the symmetrical patterned configuration we designed simplifies the manufacturing process compared with the absorbers based on the frequency superposition method. Through simulation, two obvious perfect absorption peaks at wavelengths of 22.53 and $71.98 \mu \mathrm{m}$ were found, and the absorption efficiencies were $99.74 \%$ and $99.90 \%$, respectively. Besides, a high-efficiency absorption peak at $28.61 \mu \mathrm{m}$ was also observed. Later, we studied the light absorption rate using a circle and square, respectively, under the same parameter settings. Compared with the single-band absorption metamaterial absorber proposed by Huang et al. [62], the "ancient coin"-type absorber we proposed showed two 
absorption modes. Similarly, the periodic cross-shaped graphene absorber designed by Ke et al. [63] exhibited one absorption peak with a maximum absorption of $22 \%$, while our two absorption peaks reached an absorption rate of over $99 \%$. Through a comparative analysis with these proposed structures, it could be clearly seen that our structure showed higher research value. Then, the relationship between geometric parameters of the structure and light absorption performance was explored, and the best absorption efficiency could be obtained. The controlled variable method is one of the commonly used methods in our research. By keeping the other parameters constant, the role played by different periods $P$ in light absorption was considered. With the Fermi level and relaxation time of the graphene variable, we paid attention to the adjustable characteristics of the absorber. In addition, the sensitivity of the structure to the surrounding refractive index is also a significant aspect. Finally, we discussed the influence of angle changes on TE and TM polarization. These studies indicate that our absorber has advantages of dual-band absorption, simple configuration, strong wavelength selectivity, high refractive index sensitivity, and good angular polarization tolerance, which makes it show a wide range of application prospects in biosensing, optical detection, and other fields.

\section{Materials and Methods}

The proposed monolayer graphene perfect metamaterial absorber with four unit cells is shown in Figure 1 (four periods are shown here, and our absorber can in fact be extended indefinitely as the periodic boundary condition is used). From the outside, the structure is composed of a circle and a square. The square is located at the center of the circle and is cut out. In other words, it is an "ancient coin" type, which is an ancient currency of China, consisting of a circle and a hollowed-out square in the center. The structure is composed of four layers of different materials stacked together. As a semi-infinite (i.e., $t_{1}=\infty$ ) substrate, the relative dielectric constant of the bottom silicon semiconductor layer is 3.4. The thickness of the $\mathrm{Ag}$ reflection mirror is $t_{2}=0.465 \mu \mathrm{m}$ and the relative dielectric constant is chosen as 3.7. The third insulator layer of silicon dioxide $\left(\mathrm{SiO}_{2}\right)$ separates the graphene layer from the metal layer, with thickness $t_{3}\left(t_{3}=4.1 \mu \mathrm{m}\right)$ and a relative dielectric constant of 1.97. The top layer of the structure is a periodic array of graphene disks with a square excavated, and the geometric parameters are: radius of the circle $R=1.1 \mu \mathrm{m}$; side length of the hollowed-out square $L=0.95 \mu \mathrm{m}$. In addition, the period of the proposed structure is $P x=P y=2.6 \mu \mathrm{m}$. As for graphene, the thickness of the entire graphene sheet can be replaced with the effective medium thickness $\Delta=1 \mathrm{~nm}$. Plasmon resonance occurs at the interface of the graphene and dielectric layer as doped graphene has an obvious real dielectric constant in the infrared region. When calculating the model, the thickness of graphene was set to $1 \mathrm{~nm}$, and in this case, the limit $\Delta \rightarrow 0$ was fully integrated. The whole structure was irradiated by a backward plane wave with an amplitude of 1 . The simulation time was also set to $5000 \mathrm{fs}$. Considering the boundary conditions, periodic boundary conditions were used both in the $x$ - and $y$-directions, while the $z$-direction used a perfectly matched layers (PML) boundary condition. In addition, the mesh size was set to $40 \mathrm{~nm}$ in the $x$ - and $y$-directions, and $0.2 \mathrm{~nm}$ in the $z$-direction. In practical production, the manufacturing process is as follows: The Ag film is deposited onto a silicon substrate by using the conventional electron beam evaporation method, and the $\mathrm{SiO}_{2}$ film is deposited onto the Ag layer with the same method. The top graphene layer can be grown on a copper catalyst by chemical vapor deposition, and wet-transferred to the $\mathrm{SiO}_{2}$ layer. Then, the electron beam resist is spin-coated onto the surface of the graphene, and the graphene pattern can be realized by using electron beam lithography and oxygen plasmon etching processes [64]. 


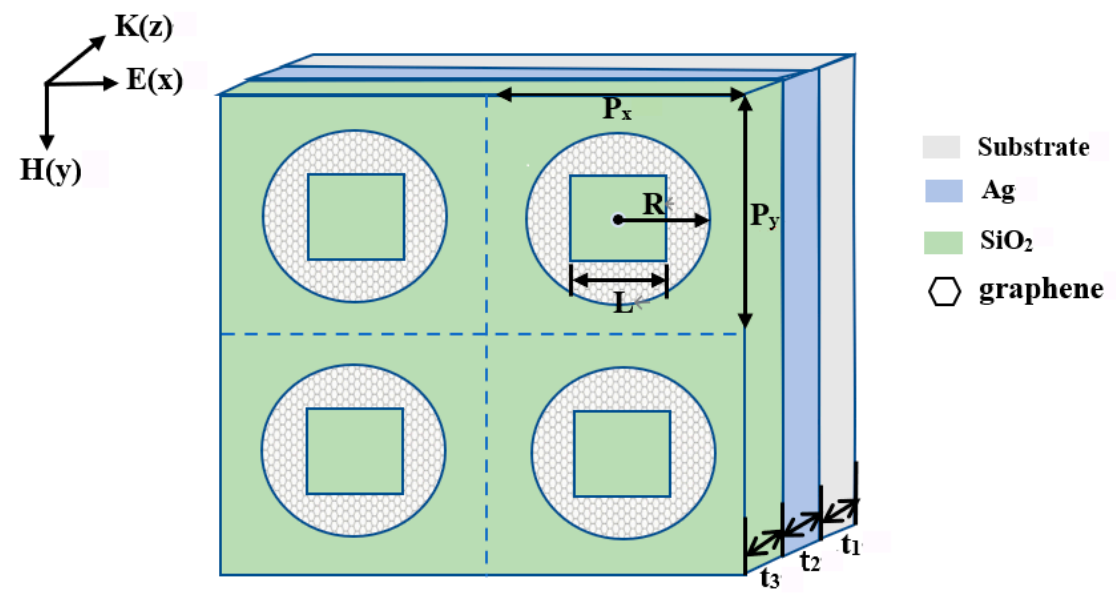

Figure 1. Three-dimensional schematic diagram of "ancient coin"-type periodic graphene perfect absorber (four units). The bottom layer is a silicon (Si) semiconductor layer as a semi-infinite (i.e., $\left.t_{1}=\infty\right)$ substrate. The thickness of the second Ag layer and the third silicon dioxide $\left(\mathrm{SiO}_{2}\right)$ layer are $t_{2}=0.465 \mu \mathrm{m}$ and $t_{3}=4.1 \mu \mathrm{m}$, respectively. The geometric parameters of the "ancient coin"-type structure are: radius of the circle $R=1.1 \mu \mathrm{m}$; side length of the hollowed-out square $L=0.95 \mu \mathrm{m}$; thickness of the graphene array of $1 \mathrm{~nm}$. The period of the proposed structure is $P=P_{x}=P_{y}=2.6 \mu \mathrm{m}$.

The law of energy conservation is a significant physical principle, and the absorption can be described by the following formula: $A$ (absorption) $=1-T$ (transmission) $-R$ (reflection). Here, the power monitor in FDTD solutions is used to monitor reflectance and transmittance. Obviously, when the transmission efficiency and reflection efficiency of the absorber are small enough, the absorption efficiency is close to the maximum value of 1. The typical skin depth is also much smaller than the thickness of the Ag layer, which hinders the transmission of electromagnetic waves, so the transmission can be suppressed, that is, $T(\lambda)=0$. In other words, the absorption depends only on reflection, which can be expressed as $A(\lambda)=1-R(\lambda)$. A key factor to ensure the coupling of low reflection and incident power is free space impedance matching, which requires continuous optimization of the structural parameters to make the absorption efficiency approximately 1 , and $R(\lambda)$ approximately 0 , to achieve perfect absorption [65].

In our simulations, graphene can be regarded as an ultra-thin dielectric layer with a dielectric constant, and its complex permittivity can be described by formula (1) [66]:

$$
\varepsilon_{g}=1+j \frac{\sigma_{g}}{\omega \varepsilon_{0} \Delta}
$$

where $\varepsilon_{0}$ represents the dielectric constant in vacuum, $\Delta$ is the thickness of the graphene layer, and $\sigma_{g}$ is the surface conductivity of graphene. According to the Kubo formula, the conductivity $\sigma_{\text {inter }}$ produced by the band transition of electrons between bands and the conductivity $\sigma_{\text {intra }}$ produced by in-band electron-photon scattering have dependences on the surface conductivity of graphene $\sigma_{g}$, which can be described as:

$$
\begin{gathered}
\sigma_{g}=\sigma_{\text {inter }}+\sigma_{\text {intra }} \\
\sigma_{\text {intra }}(\omega)=\frac{i e^{2} k_{B} T}{\pi \hbar^{2}\left(\omega+i \tau^{-1}\right)}\left(\frac{E_{f}}{k_{B} T}+2 \ln \left(e^{\frac{-E_{f}}{k_{B} T}}+1\right)\right) \\
\sigma_{\text {inter }}(\omega)=\frac{i e^{2}}{4 \pi \hbar} \ln \left|\frac{2 E_{f}-\left(\omega+i \tau^{-1}\right) \hbar}{2 E_{f}+\left(\omega+i \tau^{-1}\right) \hbar}\right|
\end{gathered}
$$

In formulas (3) and (4), $\omega$ represents the angular frequency of the incident electromagnetic wave; $E_{f}$ is the Fermi energy; and $K_{B}, \hbar$, and $T$ are the Boltzmann constant, reduced Planck constant, and room temperature (in our work, we assume $T=300 \mathrm{~K}$ ), respectively. 
Taking the highly dispersive properties of graphene into consideration, a typical implementation is invalid to capture the dispersive nature, which results in the inaccurate calculation of the quality factor. Thus, the Drude dispersion of graphene has been applied to reduce the error as an alternative measure [67]. According to the Pauli exclusion principle, $\sigma_{\text {inter }}$ can be neglected as $E_{f} \gg \hbar \omega$ in the far-infrared, and the surface conductivity of graphene $\sigma_{g}$ is only related to $\sigma_{\text {intra }}$, which is translated into the Drude-like conductance form [68]:

$$
\sigma(\omega)=\frac{i e^{2}\left|E_{f}\right|}{\pi \hbar^{2}\left(\omega+i \tau^{-1}\right)}
$$

Among them, for the relaxation time $\tau, \tau=\mu E_{f} / e v_{f}^{2}$, where $\mu$ represents the carrier mobility and the value $\mu=1.0 \mathrm{~m}^{2} \mathrm{~V}^{-1} \mathrm{~s}^{-1}$. In addition, the Fermi velocity of electrons $v_{f}=\mathrm{c} / 300 \mathrm{~m} / \mathrm{s}$. It can be seen from the formula that the surface conductivity of graphene has a close relationship with the Fermi energy and relaxation time. Combined with formula (1), the complex permittivity of graphene also depends on the chemical potential and relaxation time, which offers an ideal way to achieve the purpose of changing the properties of graphene by chemical doping or applying an external voltage.

\section{Simulation Results and Discussions}

Figure $2 \mathrm{a}-\mathrm{c}$ compare the absorption efficiency of a single disk/square structure graphene absorber and the "ancient coin"-type graphene absorber under normal incident conditions. It can be seen from Figure 2a that only one high-efficiency absorption peak appears in the single disk structure absorber, and the absorption efficiency is only $59.57 \%$. Similarly, the single square structure shown in Figure $2 b$ has only one high-efficiency absorption peak with an absorption of $76.30 \%$. As for the "ancient coin" structure we proposed, as shown in Figure 2c, it has two perfect absorption peaks at $\lambda_{1}=22.53 \mu \mathrm{m}$ and $\lambda_{2}=71.98 \mu \mathrm{m}$, and the absorption efficiency reaches $99.74 \%$ and $99.90 \%$, which is very close to $100 \%$. Therefore, it is easy to draw the conclusion that our proposed "ancient coin" structure greatly improves the absorption capacity of monolayer graphene for incident light. In order to explain this physical mechanism, we set-up separate monitors for the wavelengths corresponding to these two perfect absorption peaks. In Figure 3, the electric field distribution diagram of two peaks generated by the electric field $|\mathrm{Ez}|$ on the $x-y$ plane is given. It demonstrates that the appearance of the maximum absorption peak is associated with the electric field distribution of the graphene disk and the excavated square part. Different contributions of the square and the disk to these two peaks are portrayed from the two graphs on the right side of Figure 3. At about $22.53 \mu \mathrm{m}$, the contribution of the horizontal edge of the square to their absorption is higher than the contribution of the disk, while at about $71.98 \mu \mathrm{m}$, the contribution of the edge of the graphene nanodisk is largest, and the square hardly contributes. From this, we can reach the conclusion that different resonance bands have different resonance regions.

The absorption bandwidth can be defined by the half-height width (also called FWHM), which refers to the corresponding spectral width when the peak height of the absorption band is half. The FWHMs of mode $\lambda_{1}$ and mode $\lambda_{2}$ are 0.96 and 0.99 , respectively, through calculations. The absorption bandwidth is relatively narrow, which means that our absorber has a strong wavelength selectivity. In addition, the quality factor $Q$ of the proposed structure was studied. It is a physical quantity that represents the relative bandwidth of the resonator's vibration frequency. The higher the quality factor, the narrower the frequency range that can generate resonance, the longer the duration, and the better the stability. The quality factor $Q$ can be obtained by the formula $Q=\lambda /$ FWHM [69]. In our absorber, the $Q$ factor of mode $\lambda_{2}$ is 72.28, which is 3.07 times the $Q$ factor of mode $\lambda_{1}$, and it demonstrates that mode $\lambda_{2}$ has more potential for application in the field of sensor than $\lambda_{1}$. 
(a)

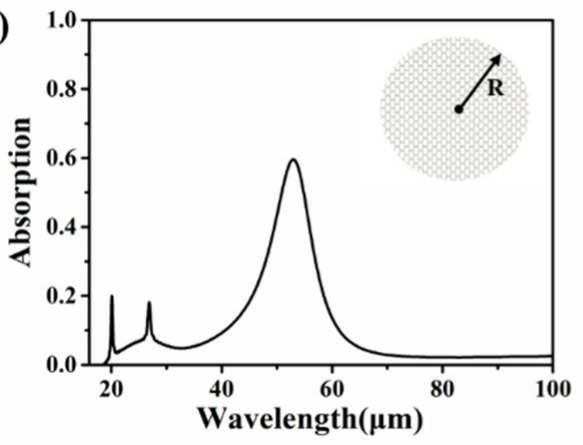

(b)

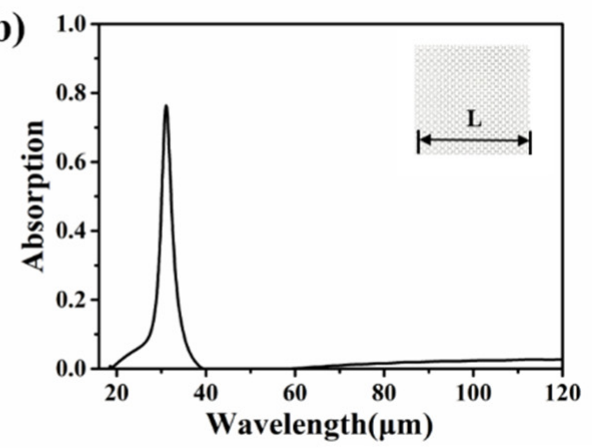

(c)

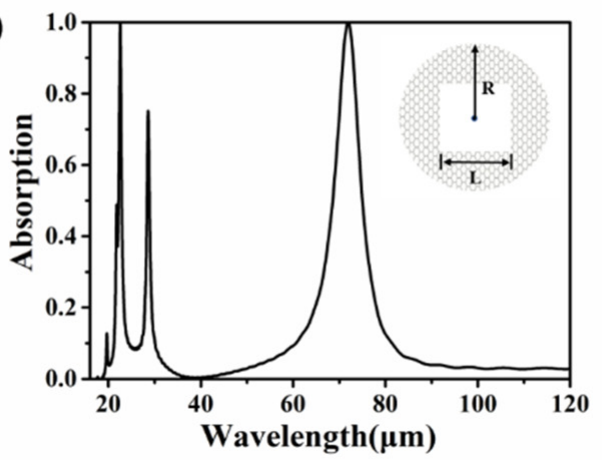

Figure 2. (a) A schematic diagram of light absorption of a single-disc-structure graphene absorber and a planform of the absorber, with radius $R$ and period $P$. (b) A schematic diagram of light absorption of a single-square-structure graphene absorber and a planform of the absorber, with side length $L$ and period P. (c) The light absorption diagram and planform of the "ancient coin" graphene absorber.

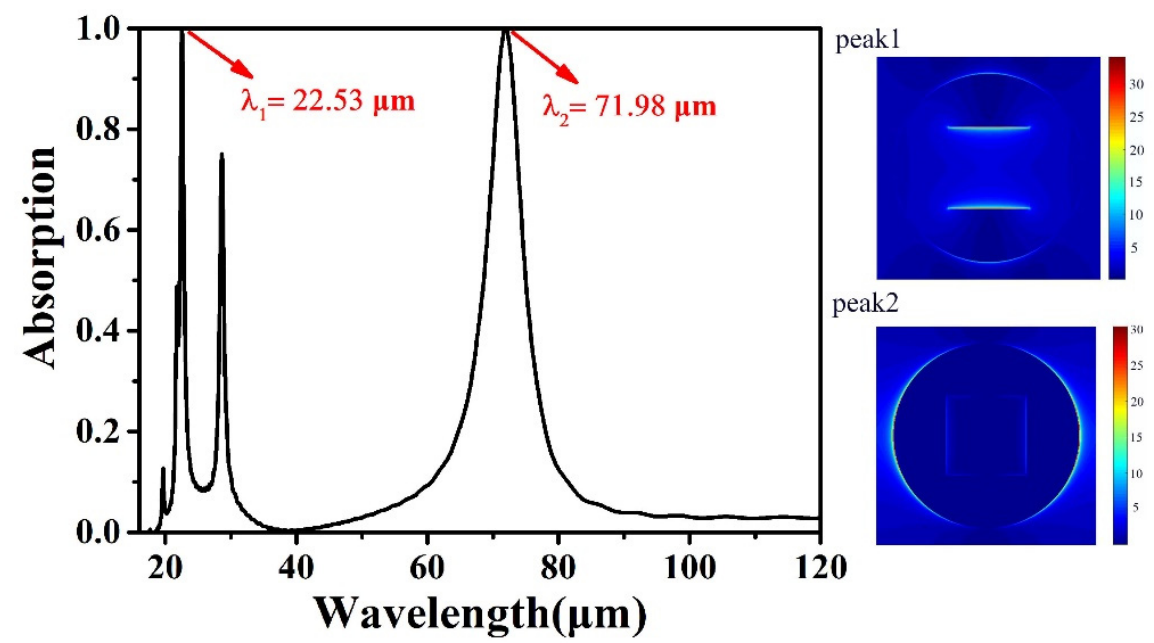

Figure 3. The optical absorption spectrum of the "ancient coin" structure and the corresponding electric field diagrams of the two perfect absorption peaks on the $x-y$ plane.

Based on previous studies [63,70], we found that the absorptive capacity is closely relevant to the period $P$. Thus, in this part, we studied the relationship between the period $P$ and the light absorption rate. Figure 4 suggests that different periods $P$ show different changing trends of the two perfect absorption peaks. For mode $\lambda_{1}$, as the period increases from 2.4 to $3.2 \mu \mathrm{m}$ (each interval of $0.2 \mu \mathrm{m}$ takes a value), the absorption efficiency increases first and then decreases to $86.26 \%$. When $P=2.6 \mu \mathrm{m}$, the absorption efficiency reaches the maximum value of $99.74 \%$, so the period was set to $2.6 \mu \mathrm{m}$ in our work. In addition, the wavelength of the absorption peak remains almost unchanged with the increase in $P$, which is basically located at $\lambda_{1}=22.53 \mu \mathrm{m}$. This is because the resonance condition remains 
almost the same level, and the resonance peak wavelength changes slightly in a small range due to the influence of resonance caused by the horizontal edge of the square. For mode $\lambda_{2}$, with the increase in period, the absorption efficiency is basically maintained at around $99 \%$ with hardly any fluctuation, while the absorption peak shows an obvious blueshift from 74.31 to $64.95 \mu \mathrm{m}$. The reason is that when the structure is illuminated by a plane wave with the electric field along the $x$ - direction, the effective index of graphene surface plasmon polaritons along the $x$ - direction decreases as $P$ increases. The resonance wavelength also shifts to the shorter band as the wavelength is proportional to the effective index.

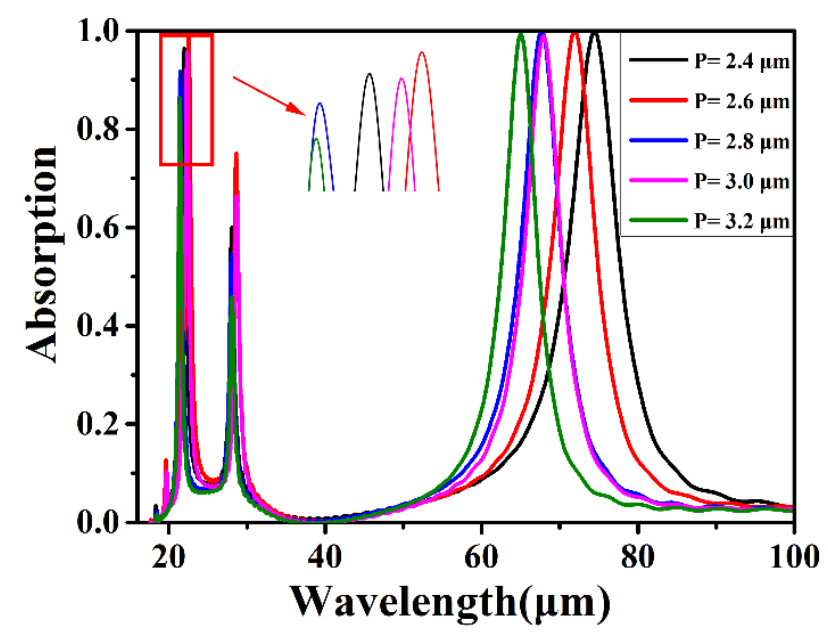

Figure 4. Absorption spectrum of "ancient coin"-type absorber under different periods $P$.

After that, the influence of the geometrical parameters of our proposed structure on the absorption rate attracted our attention, as shown in Figure 5. In Figure 5a, the curves of different color represent the absorption spectra of the absorber with different disk radii R. Here, the controlled variable method was used, with $P=2.6 \mu \mathrm{m}$ and the side length of square $L=0.95 \mu \mathrm{m}$ fixed. By increasing the value of $\mathrm{R}$ regularly, we explored the influence of disk radius on the overall absorption. It is apparent from Figure 5a that as $R$ increases from 0.8 to $1.2 \mu \mathrm{m}$, the wavelengths of mode $\lambda_{1}$ and mode $\lambda_{2}$ experiences a blueshift first, and then a redshift, which shows that both modes are sensitive to the changes in $R$. Moreover, the absorption efficiency of two peaks shows the same trends of increasing first and then decreasing with the gradual increase in $R$. It is the increase in the ratio filled with graphene as the disk radius $R$ increases that results in the enhancement of absorption capacity. However, when $R$ is too large (over $1.1 \mu \mathrm{m}$ ), the absorption efficiency decreases as the structure is close to the adjacent graphene pattern units, and it can interact with each other.
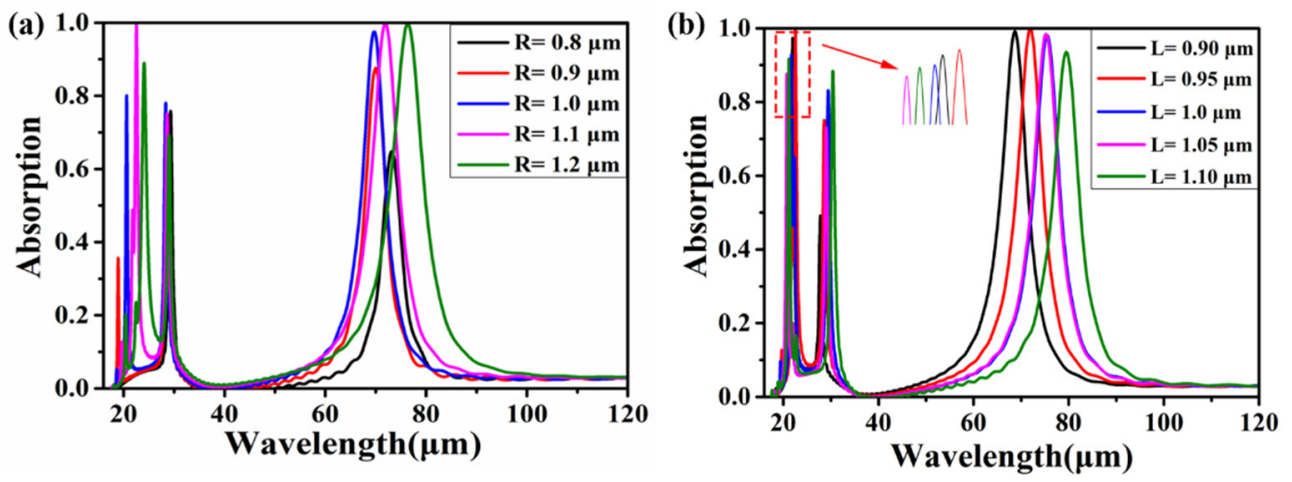

Figure 5. (a) The relationship between the radius $R$ of the disc and the light absorption rate. (b) The relationship between the side length $L$ of the square and the light absorption. 
Figure $5 b$ illustrates the effect of the size of the hollowed-out square on the light absorption rate with period $P$ and disk radius $R$ constant. It can also be seen that as $L$ increases from 0.90 to $1.10 \mu \mathrm{m}$ in intervals of $0.05 \mu \mathrm{m}$, the resonance wavelength of mode $\lambda_{1}$ changes slightly in a small range; this is the same physical mechanism as when $P$ increases. However, the mode $\lambda_{2}$ shows a redshift from 68.66 to $79.46 \mu \mathrm{m}$, which can be attributed to the increase in the effective index of graphene nanodisk surface plasmon polaritons due to the tighter distribution of electric fields. At the same time, the absorption efficiency of mode $\lambda_{1}$ and mode $\lambda_{2}$ increases first and then decreases. This is because, as the area of the square excavated increases, the internal arrangement of graphene becomes tighter, and the increased surface plasmon polaritons can lead to an increase in absorption efficiency to light. However, when $L>0.95 \mu \mathrm{m}$, the resonance peak excited between different regions interfere with each other, resulting in a reduction in the absorption efficiency. Through simulation, it was found that when $R=1.10 \mu \mathrm{m}$ and $L=0.95 \mu \mathrm{m}$, the maximum absorption of two peaks can be obtained.

In Figure $6 a$, we explored the absorption performance on $E_{f}$ of graphene. Here, the other geometric parameters we took are also consistent with Figure 1. Based on the existing literature, we learned that the concept of perfect absorption comes from electric dipole resonance and magnetic dipole resonance [71]. As $E_{f}$ increases from 0.4 to $0.8 \mathrm{eV}$, the wavelengths of the two resonance peaks blueshift. The graphene resonator can be treated as a dipole antenna as the wavelength of incident light is much larger than the structural parameters of the model. Thus, the effective index of graphene surface plasmons decreases with $E_{f}$, resulting in a decline in the resonance wavelength. The absorption efficiency of the absorption peak shows a trend of first increasing and then decreasing. When $E_{f}=0.6 \mathrm{eV}$, both absorption peaks reach the maximum absorption efficiency. Therefore, in this paper, the $E_{f}$ of graphene was set as $0.6 \mathrm{eV}$.
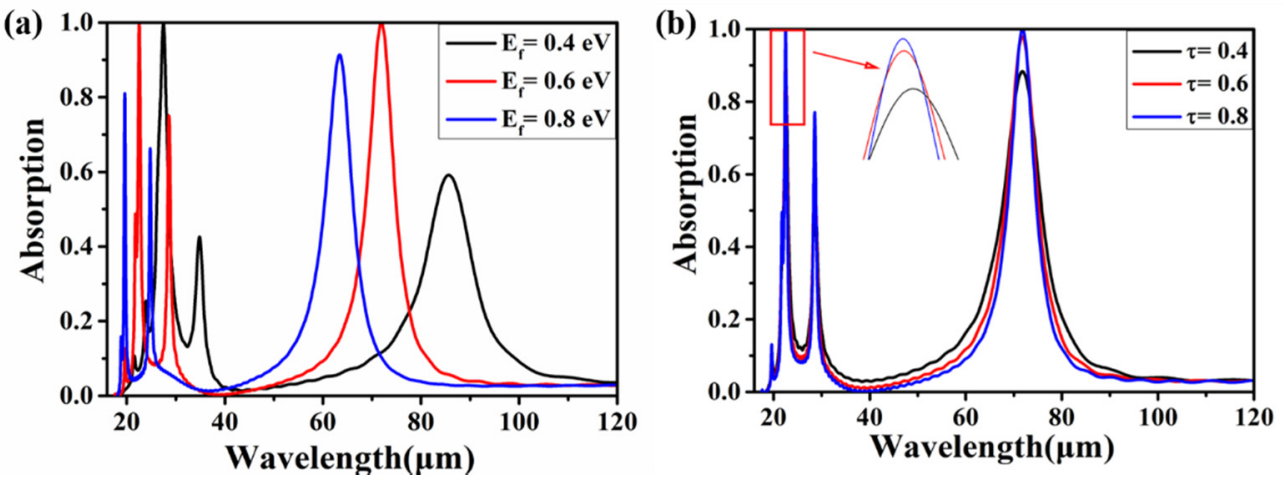

Figure 6. (a) Absorption spectra corresponding to different Fermi levels $E_{f}$. (b) Absorption spectra corresponding to different relaxation times.

In Figure $6 \mathrm{~b}$, we discuss the influence of different relaxation times on the absorption rate, and the other parameters are the same as we used in Figure 1. The relaxation time of electrons in graphene can be calculated with the formula: $\tau=\left(\mu \cdot \mu_{c}\right) /\left(e \cdot v_{F}^{2}\right)$, where $e$ is the electron charge, $v_{F}=10^{6} \mathrm{~m} / \mathrm{s}$ is the Fermi velocity, $\mu_{c}=0.6 \mathrm{eV}$ is the chemical potential, and $\mu$ is the carrier mobility. We can learn from the above formula that the relaxation time will increase with $\mu$. Thus, in the simulation debugging, the relaxation time can be changed with the method of setting different $\mu$. The result in this figure demonstrates that the width of the peak gradually narrows and the absorption rate gradually increases with the relaxation time. This is mainly because as $\tau$ increases, the plasmon oscillations absorption will increase with the contribution of charge carriers, leading to a higher absorption to light. When $\tau=0.8 \mathrm{ps}$, the two absorption peaks both reach the maximum absorption value of over $99 \%$. Thus, in this paper, the relaxation time was set to 0.8 ps.

Next, we studied the relationship between background refractive index (RI) and absorption rate. From Figure 7a, when RI increases from 1.0 to 1.5 (with an interval of 
0.1 ), both the wavelengths corresponding to the two perfect absorption peaks redshift. Regarding the change in absorbance shown in Figure 7c, the result demonstrates that the absorption rate of the first peak decreases with RI, but the second absorption peak remains the same. In addition, the specific condition of the redshift in the wavelength is shown in Figure $7 \mathrm{~b}$. The result indicates that the resonance wavelengths corresponding to the two perfect absorption peaks linearly increase with the RI. It is clear from Figure $7 \mathrm{~b}$ that the slope of the line corresponding to the wavelength $\lambda_{2}$ is greater than the line corresponding to the wavelength $\lambda_{1}$; therefore, the wavelength sensitivity of the perfect absorption peak $\lambda_{2}$ is higher than $\lambda_{1}$ as the slope can be used to express the wavelength sensitivity. The following formula can also be used to calculate the wavelength sensitivity of the two absorption peaks:

$$
S=\frac{\text { Peakshift }(e v)}{\Delta R I U}
$$

(a)
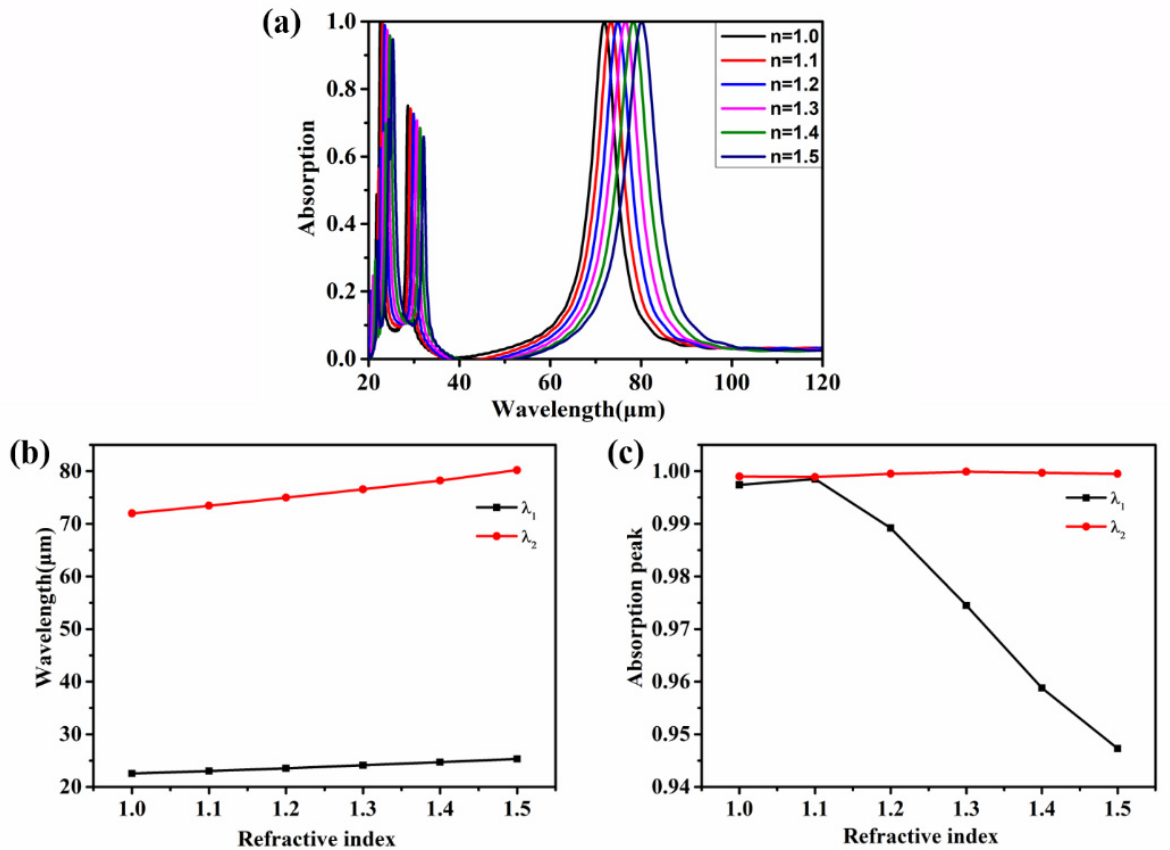

Figure 7. (a) Absorption spectra under different background refractive indices. (b) The function relationship between the wavelengths of two perfect absorption peaks and the background refractive index. (c) The relationship between the absorption rate of two perfect absorption peaks and the background refractive index.

This formula represents the ratio of shift values of the peak to the changes in RI. By entering the values, we can calculate that the sensitivity of wavelength $\lambda_{1}$ is $5.6 \mu \mathrm{m} / \mathrm{RIU}$, while the sensitivity of wavelength $\lambda_{2}$ is $16.46 \mu \mathrm{m} / \mathrm{RIU}$. The result is also consistent with the performance of the slope in Figure $7 \mathrm{~b}$, and the two figures can confirm each other. According to this property, we can see that the absorber structure we proposed has potential application value in sensors.

Finally, we analyzed the relationship between the angle and absorptive capacity of the absorber by adjusting the angle of incident light. Figure 8 shows the absorption condition of our proposed absorber structure under two incident light polarization modes (TM polarization and TE polarization). In Figure 8a, we set the polarization direction (angle phi) to 0 and gradually increased the value of $\theta$ from 0 to $30^{\circ}$ to obtain the absorption spectra generated by the incident light at different angles under TM polarization. In Figure 8b, we set the polarization direction to $90^{\circ}$ and took the same steps under TE polarization. We found that the half-height width, the wavelength of the resonant peak, and the maximum absorption efficiency shown in Figure 8a,b remained the same. It is the local plasmon resonance on the patterned graphene layer that results in the appearance of resonant peaks. 
The wavelength of the surface plasmons of the graphene nanostructure is also shorter than the vacuum wavelength of the incident light [72], for which reason the two resonant modes remained unchanged. The result demonstrates that the proposed structure does not depend on the polarization of incident light, which means the structure is not sensitive to the TM and TE polarization modes. In addition, Figure $8 \mathrm{c}$ provides evidence for this phenomenon. We scanned the polarization angle from $0^{\circ}$ to $50^{\circ}$, and we added the reflectivity monitor and transmissivity monitor to detect absorption conditions. The two red bands represent two high absorption peaks. When the polarization angle increased from 0 to $50^{\circ}$, the center frequencies of the two high absorption bands did not change, which indicates that the structure is not sensitive to the polarization angle of light. The phenomenon can be attributed to the high symmetry of our proposed structure, which eliminates the internal polarization angle dependence. Therefore, the proposed absorber can be promising in many integrated optical devices.
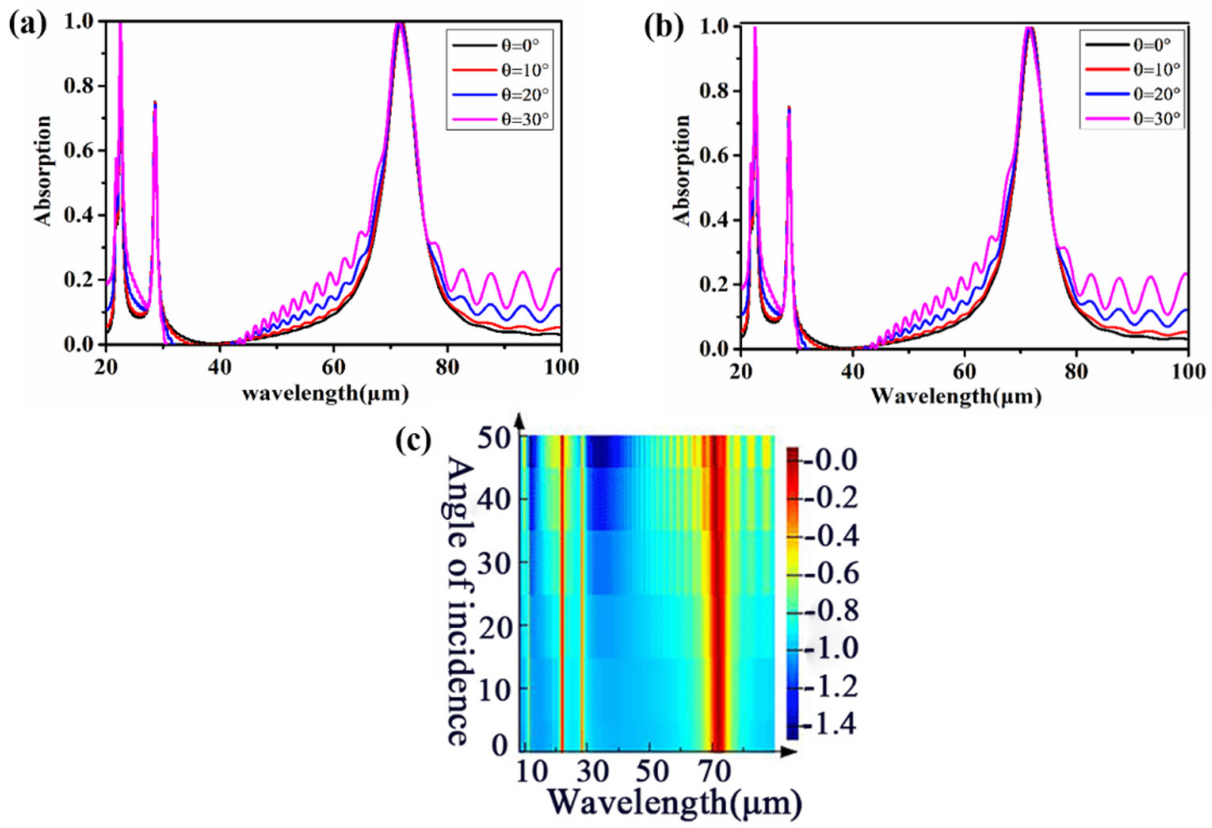

Figure 8. (a) The generated spectra at different incident angles of light under TM polarization. (b) The generated spectra at different incident angles of light under TE polarization. (c) Scanning graph of the absorbance of the two peaks at different polarization azimuths.

\section{Conclusions}

An "ancient coin"-type dual-band tunable graphene metamaterial perfect absorber is proposed in this paper. By adjusting the geometric parameters of the structure, the Fermi level, and the relaxation time of the graphene, two continuously adjustable perfect absorption peaks were obtained, which reached an absorption rate of over $99 \%$ at 22.53 and $71.98 \mu \mathrm{m}$. In addition, by investigating the background refractive index of the "ancient coin" structure, the result indicated that the maximum absorption efficiency corresponding to wavelength $\lambda_{1}$ decreased with the refractive index, while the maximum absorption efficiency corresponding to wavelength $\lambda_{2}$ had little change. At the same time, the sensitivities of wavelength $\lambda_{1}$ and wavelength $\lambda_{2}$ were 5.6 and $16.46 \mu \mathrm{m} /$ RIU, respectively. Finally, we changed the polarization direction and incident angle, and we found that the two perfect absorption peaks of the absorber always remained in the state of maximum absorption, that is, our structure has good angular polarization tolerance. Compared with previous devices, our structure has broad application prospects in biosensing, photoelectric detection, and other fields due to its good refractive index sensitivity, good angular polarization tolerance, and highly symmetrical configuration. 
Author Contributions: Conceptualization, H.L. and Q.S.; data curation, H.L., Q.S. and Y.Y. (Yinting Yi); formal analysis, Y.Y. (Yougen Yi) and Z.L.; methodology, H.L., Q.S., Y.Y. (Yinting Yi), Y.Y. (Yougen Yi) and Z.L.; resources, Y.Y. (Yougen Yi); software, Y.Y. (Yougen Yi), S.C. and Z.L.; data curation, H.L.; writing-original draft preparation, H.L.; writing-review and editing, H.L. and Y.Y. (Yougen Yi). All authors have read and agreed to the published version of the manuscript.

Funding: The authors are grateful to the support by the National Natural Science Foundation of China (NSFC) (11904032, 11774054, 12075036), the Scientific Research Plan of the Provincial Education Department in Hubei (Q20161309), and the Natural Science Foundation of Hunan Province (2019JJ50133).

Institutional Review Board Statement: Not applicable.

Informed Consent Statement: Not applicable.

Data Availability Statement: Publicly available datasets were analyzed in this study. This data can be found here: [https://www.lumerical.com/], accessed on 1 January 2021.

Conflicts of Interest: The authors declare no conflict of interest.

\section{References}

1. Walser, R.M. Electromagnetic metamaterials. Complex Mediums II: Beyond Linear Isotropic Dielectrics; International Society for Optics and Photonics: Bellingham, WA, USA, 2001; Volume 4467, pp. 1-16. [CrossRef]

2. Chen, Z.; Chen, H.; Jile, H.; Xu, D.; Yi, Z.; Lei, Y.; Chen, X.; Zhou, Z.; Cai, S.; Li, G. Multi-band multi-tunable perfect plasmon absorber based on L-shaped and double-elliptical graphene stacks. Diam. Relat. Mater. 2021, 115, 108374. [CrossRef]

3. Liu, Z.; Zhang, X.; Zhou, F.; Luo, X.; Zhang, Z.; Qin, Y.; Zhuo, S.; Gao, E.; Li, H.; Yi, Z. Triple plasmon-induced transparency and optical switch desensitized to polarized light based on a mono-layer metamaterial. Opt. Express 2021, 29, 13949-13959. [CrossRef] [PubMed]

4. Cheng, T.; Gao, W.; Gao, H.; Wang, S.; Yi, Z.; Wang, X.; Yang, H. Piezocatalytic degradation of methylene blue, tetrabromobisphenol $\mathrm{A}$ and tetracycline hydrochloride using $\mathrm{Bi}_{4} \mathrm{Ti}_{3} \mathrm{O}_{12}$ with different morphologies. Mater. Res. Bull. 2021, 141, 111350. [CrossRef]

5. He, Z.; Li, L.; Ma, H.; Pu, L.; Xu, H.; Yi, Z.; Cao, X.; Cui, W. Graphene-based metasurface sensing applications in terahertz band. Results Phys. 2021, 21, 103795. [CrossRef]

6. An, S.; Lv, J.; Yi, Z.; Liu, C.; Yang, L.; Wang, F.; Liu, Q.; Su, W.; Li, X.; Sun, T.; et al. Ultra-short and dual-core photonic crystal fiber polarization splitter composed of metal and gallium arsenide. Optik 2021, 226, 165779. [CrossRef]

7. Wang, X.X.; Zhu, J.K.; Xu, Y.Q.; Qi, Y.P.; Zhang, L.P.; Yang, H.; Yi, Z. A novel plasmonic refractive index sensor based on gold/silicon complementary grating structure. Chin. Phys. B 2021, 30, 024207. [CrossRef]

8. Wang, Y.; Yi, Y.; Xu, D.; Yi, Z.; Li, Z.; Chen, X.; Jile, H.; Zhang, J.; Zeng, L.; Li, G. Terahertz tunable three band narrowband perfect absorber based on Dirac semimetal. Phys. E Low-Dimens. Syst. Nanostruct. 2021, 131, 114750. [CrossRef]

9. Qi, Y.; Zhang, B.; Ding, J.; Zhang, T.; Wang, X.; Yi, Z. Efficient Manipulation of Terahertz waves by multi-bit Coding Metasurfaces and its further application. Chin. Phys. B 2021, 30, 024211. [CrossRef]

10. Li, J.; Chen, X.; Yi, Z.; Yang, H.; Tang, Y.; Yi, Y.; Yao, W.; Wang, J.; Yi, Y. Broadband solar energy absorber based on monolayer molybdenum disulfide using tungsten elliptical arrays. Mater. Today Energy 2020, 16, 100390. [CrossRef]

11. Wang, X.X.; Tong, H.; Pang, Z.Y.; Zhu, J.K.; Wu, X.X.; Yang, H.; Qi, Y. Theoretical realization of three-dimensional nanolattice structure fabrication based on high-order waveguide-mode interference and sample rotation. Opt. Quant. Electron. 2019, 51, 38. [CrossRef]

12. Chen, Z.; Chen, H.; Yin, J.; Zhang, R.; Jile, H.; Xu, D.; Yi, Z.; Zhou, Z.; Cai, S.; Yan, P. Multi-band, Tunable, High Figure of Merit, High Sensitivity Single-layer Patterned Graphene-Perfect Absorber Based On Surface Plasmon Resonance. Diam. Relat. Mater. 2021, 116, 108393. [CrossRef]

13. Deng, Y.; Cao, G.T.; Yang, H.; Zhou, X.Q.; Wu, Y.W. Dynamic Control of Double Plasmon-Induced Transparencies in ApertureCoupled Waveguide-Cavity System. Plasmonics 2018, 13, 345-352. [CrossRef]

14. Long, F.; Zhang, Z.H.; Wang, J.; Yan, L.; Zhou, B.W. Cobalt-nickel bimetallic nanoparticles decorated graphene sensitized imprinted electrochemical sensor for determination of octylphenol. Electrochim. Acta 2015, 168, 337-345. [CrossRef]

15. Yao, J.; Liu, Z.; Liu, Y.; Wang, Y.; Sun, C.; Bartal, G.; Stacy, A.M.; Zhang, X. Optical negative refraction in bulk metamaterials of nanowires. Science 2008, 321, 930. [CrossRef] [PubMed]

16. Zhang, Z.H.; Cai, R.; Long, F.; Wang, J. Development and application of tetrabromobisphenol A imprinted electrochemical sensor based on graphene/carbon nanotubes three-dimensional nanocomposites modified carbon electrode. Talanta 2015, 134, 435-442. [CrossRef] [PubMed]

17. Chen, H.J.; Zhang, Z.H.; Cai, R.; Kong, X.Q.; Chen, X.; Liu, Y.N.; Yao, S.Z. Molecularly imprinted electrochemical sensor based on a reduced graphene modified carbon electrode for tetrabromobisphenol A detection. Analyst 2013, 138, 2769-2776. [CrossRef]

18. Zhang, Y.; Yi, Z.; Wang, X.; Chu, P.; Yao, W.; Zhou, Z.; Cheng, S.; Liu, Z.; Wu, P.; Pan, M.; et al. Dual band visible metamaterial absorbers based on four identical ring patches. Phys. E Low-Dimens. Syst. Nanostruct. 2021, 127, 114526. [CrossRef] 
19. Chen, X.; Wu, W.; Zhang, W.; Wang, Z.; Fu, Z.; Zhou, L.; Yi, Z.; Li, G.; Zeng, L. Blue and green double band luminescent carbon quantum dots: Synthesis, origin of photoluminescence, and application in white light-emitting devices. Appl. Phys. Lett. 2021, 118, 153102. [CrossRef]

20. Yi, Z.; Li, J.K.; Lin, J.C.; Qin, F.; Chen, X.F.; Yao, W.T.; Liu, Z.M.; Cheng, S.B.; Wu, P.H.; Li, H.L. Broadband polarization-insensitive and wide-angle solar energy absorber based on tungsten ring-disc array. Nanoscale 2020, 12, 23077-23083. [CrossRef]

21. Cheng, Z.H.; Liao, J.; He, B.Z.; Zhang, F.; Zhang, F.A.; Huang, X.H.; Zhou, L. One-Step Fabrication of Graphene Oxide Enhanced Magnetic Composite Gel for Highly Efficient Dye Adsorption and Catalysis. ACS Sustain. Chem. Eng. 2015, 3, 1677-1685. [CrossRef]

22. Landy, N.I.; Sajuyigbe, S.; Mock, J.J.; Smith, D.R.; Padilla, W.J. Perfect metamaterial absorber. Phys. Rev. Lett. 2008, 100, 207402. [CrossRef]

23. Chu, P.X.; Chen, J.X.; Xiong, Z.G.; Yi, Z. Controllable frequency conversion in the coupled time-modulated cavities with phase delay. Opt. Commun. 2020, 476, 126338. [CrossRef]

24. Cai, R.; Rao, W.; Zhang, Z.H.; Long, F.; Yin, Y.L. An imprinted electrochemical sensor for bisphenol A determination based on electrodeposition of a graphene and Ag nanoparticle modified carbon electrode. Anal. Methods 2014, 6, 1590-1597. [CrossRef]

25. Jiang, L.Y.; Yi, Y.T.; Yi, Z.; Yang, H.; Li, Z.Y.; Su, J.; Zhou, Z.G.; Chen, X.F.; Yi, Y.G. A four-band perfect absorber based on high quality factor and high figure of merit of monolayer molybdenum disulfide. Acta Phys. Sin. 2021, 70, 128101. [CrossRef]

26. Pan, M.; Huang, H.Z.; Chen, W.Z.; Li, S.; Xie, Q.; Xu, F.; Wei, D.; Fang, J.; Fan, B.; Cai, L. Design of narrow-band absorber based on symmetric silicon grating and research on its sensing performance. Coatings 2021, 11, 553. [CrossRef]

27. Xu, L.; Zeng, J.; Luo, X.; Xia, L.; Ma, Z.; Peng, B.; Li, Z.; Zhai, X.; Wang, L. Dual-Band Plasmonic Perfect Absorber Based on the Hybrid Halide Perovskite in the Communication Regime. Coatings 2021, 11, 67. [CrossRef]

28. Liu, X.; Tyler, T.; Starr, T.; Starr, A.F.; Jokerst, N.M.; Padilla, W.J. Taming the blackbody with infrared metamaterials as selective thermal emitters. Phys. Rev. Lett. 2011, 107, 045901. [CrossRef] [PubMed]

29. Zhang, X.; Liu, Z.; Zhang, Z.; Qin, Y.; Zhuo, S.; Luo, X.; Zhou, F.; Yi, Z.; Wang, J.; Wang, Y. Triple plasmon-induced transparency in graphene and metal metamaterials and its anomalous property. J. Phys. D Appl. Phys. 2021, 54, 284001. [CrossRef]

30. Chen, H.; O’Hara, J.F.; Azad, A.K.; Taylor, A.J.; Averitt, R.D. Experimental demonstration of frequency-agile terahertz metamaterials. Nat. Photonics 2008, 2, 295-298. [CrossRef]

31. Zhang, Z.; Liu, Z.; Zhou, F.; Wang, J.; Wang, Y.; Zhang, X.; Qin, Y.; Zhuo, S.; Luo, X.; Gao, E.; et al. Broadband plasmon-induced transparency modulator in the terahertz band based on multilayer graphene metamaterials. JOSA A 2021, 38, 784-789. [CrossRef]

32. Deng, Y.; Cao, G.; Wu, Y.; Zhou, X.; Liao, W. Theoretical Description of Dynamic Transmission Characteristics in MDM Waveguide Aperture-Side-Coupled with Ring Cavity. Plasmonics 2015, 10, 1537-1543. [CrossRef]

33. Cao, G.; Li, H.; Deng, Y.; Zhan, S.; He, Z.; Li, B. Systematic Theoretical Analysis of Selective-Mode Plasmonic Filter Based on Aperture-Side-Coupled Slot Cavity. Plasmonics 2014, 9, 1163-1169. [CrossRef]

34. Driscoll, T.; Palit, S.; Qazilbash, M.M.; Brehm, M.; Keilmann, F.; Chae, B.-G.; Yun, S.-J.; Kim, H.T.; Cho, S.-Y.; Jokerst, N.M.; et al. Dynamic tuning of an infrared hybrid-metamaterial resonance using vanadium dioxide. Appl. Phys. Lett. 2008, 93, 18. [CrossRef]

35. Novoselov, K.S.; Fal'ko, V.I.; Colombo, L.; Gellert, P.R.; Kim, K.A. A roadmap for graphene. Nature 2012, 490, 192-200. [CrossRef]

36. Lin, X.; Li, Y.J.; Chen, F.T.; Xu, P.; Li, M. Facile synthesis of mesoporous titanium dioxide doped by Ag-coated graphene with enhanced visible-light photocatalytic performance for methylene blue degradation. RSC Adv. 2017, 7, 25314-25324. [CrossRef]

37. Tang, N.M.; Li, Y.J.; Chen, F.T.; Han, Z.Y. In situ fabrication of a direct Z-scheme photocatalyst by immobilizing CdS quantum dots in the channels of graphene-hybridized and supported mesoporous titanium nanocrystals for high photocatalytic performance under visible light. RSC Adv. 2018, 8, 42233-42245. [CrossRef]

38. Yang, X.; Zhang, F.; Hu, Y.J.; Chen, D.Z.; He, Z.Q.; Xiong, L.Z. Gold Nanoparticals Doping Graphene Sheets Nanocomposites Sensitized Screen-printed Carbon Electrode as a Disposable Platform for Voltammetric Determination of Guaiacol in Bamboo Juice. Int. J. Electrochem. Sci. 2014, 9, 5061-5072.

39. Koppens, F.H.L.; Chang, D.E.; Thongrattanasiri, S.; de Abajo, F.J.G. Graphene plasmonics: A platform for strong light-matter interactions. Nano Lett. 2011, 11, 3370-3377. [CrossRef] [PubMed]

40. Christensen, J.; Manjavacas, A.; Thongrattanasiri, S.; Koppens, F.H.H.; de Abajo, F.J.G. Graphene plasmon waveguiding and hybridization in individual and paired nanoribbons. ACS Nano 2012, 6, 431-440. [CrossRef]

41. Long, F.; Zhang, Z.H.; Wang, J.; Yan, L.; Lu, P.P.; Yang, Z.X. Magnetic Graphene Modified Imprinted Electrochemical Sensor for Detection of 4-Octylphenol. Chin. J. Anal. Chem. 2016, 44, 908-914. [CrossRef]

42. Zhou, Q.; Ju, W.; Yong, Y.; Zhang, Q.; Liu, Y.; Li, J. Effect of the N/P and transition-metal co-doping on the quantum capacitance of supercapacitor electrodes based on mono- and multilayer graphene. Carbon 2020, 170, 368-379. [CrossRef]

43. Cen, C.L.; Zhang, Y.B.; Chen, X.F.; Yang, H.; Yi, Z.; Yao, W.T.; Tang, Y.J.; Yi, Y.G.; Wang, J.Q.; Wu, P.H. A dual-band metamaterial absorber for graphene surface plasmon resonance at terahertz frequency. Phys. E 2020, 117, 113840. [CrossRef]

44. Wang, X.; Duan, J.; Chen, W.; Zhou, C.; Liu, T.; Xiao, S. Controlling light absorption of graphene at critical coupling through magnetic dipole quasi-bound states in the continuum resonance. Phys. Rev. B 2021, 102, 155432. [CrossRef]

45. Chen, J.; Nie, H.; Peng, C.; Qi, S.B.; Tang, C.J.; Zhang, Y.; Wang, L.H.; Park, G.S. Enhancing the Magnetic Plasmon Resonance of three-dimensional optical Metamaterials via Strong Coupling for High-Sensitivity Sensing. J. Light. Technol. 2018, $36,3481$. [CrossRef]

46. Jiang, L.; Yuan, C.; Li, Z.; Su, J.; Yi, Z.; Yao, W.; Wu, P.; Liu, Z.; Cheng, S.; Pan, M. Multi-band and high-sensitivity perfect absorber based on monolayer grapheme metamaterial. Diam. Relat. Mater. 2021, 111, 108227. [CrossRef] 
47. Li, J.H.; Jiang, J.B.; Zhao, D.; Xu, Z.F.; Liu, M.Q.; Liu, X.; Tong, H.X.; Qian, D. Novel hierarchical sea urchin-like Prussian blue@palladium core-shell heterostructures supported on nitrogen-doped reduced graphene oxide: Facile synthesis and excellent guanine sensing performance. Electrochim. Acta 2020, 330, 135196. [CrossRef]

48. Cai, L.; Zhang, Z.H.; Xiao, H.M.; Chen, S.; Fu, J.L. An eco-friendly imprinted polymer based on graphene quantum dots for fluorescent detection of p-nitroaniline. RSC Adv. 2019, 9, 41383-41391. [CrossRef]

49. Yu, P.; Yang, H.; Chen, X.; Yi, Z.; Yao, W.; Chen, J.; Yi, Y.; Wu, P. Ultra-wideband solar absorber based on refractory titanium metal. Renew. Energy 2020, 158, 227-235. [CrossRef]

50. Yang, M.; Kong, Q.; Feng, W.; Yao, W. N/O double-doped biomass hard carbon material realizes fast and stable potassium ion storage. Carbon 2021, 176, 71-82. [CrossRef]

51. He, Z.; Li, L.; Cui, W.; Wang, Y.; Xue, W.; Xu, H.; Yi, Z.; Li, C.; Li, Z. Unidirectional reflectionless propagation of near-infrared light in resonator-assisted non-parity-time symmetric waveguides. New J. Phys. 2021, 23, 053015. [CrossRef]

52. Zhang, B.; Qi, Y.; Zhang, T.; Zhang, Y.; Liu, W.; Wang, L.; Ding, J.; Wang, X.; Yi, Z. Tunable multi-band terahertz absorber based on composite graphene structures with square ring and Jerusalem cross. Results Phys. 2021, 25, 104233. [CrossRef]

53. Zhang, X.; Liu, Z.; Zhang, Z.; Gao, E.; Luo, X.; Zhou, F.; Li, H.; Yi, Z. Polarization-sensitive triple plasmon-induced transparency with synchronous and asynchronous switching based on monolayer graphene metamaterials. Opt. Express 2020, 28, 36771-36783. [CrossRef]

54. Wang, Y.; Cui, W.; Ma, H.; Xu, H.; Yi, Z.; Cao, X.; Ren, X.; He, Z. Outstanding slow-light effect for graphene metasurface in terahertz. Results Phys. 2021, 23, 104002. [CrossRef]

55. Li, J.H.; Jiang, J.B.; Xu, Z.F.; Liu, M.Q.; Tang, S.P.; Yang, C.M.; Qian, D. Facile synthesis of Ag@Cu ${ }_{2} \mathrm{O}$ heterogeneous nanocrystals decorated N-doped reduced graphene oxide with enhanced electrocatalytic activity for ultrasensitive detection of $\mathrm{H}_{2} \mathrm{O}_{2}$. Sens. Actuators B Chem. 2018, 260, 529-540. [CrossRef]

56. Zhou, Q.; Ju, W.; Yong, Y.; Liu, Y.; Li, J. Quantum capacitance of supercapacitor electrodes based on germanene influenced by vacancy and co-doping: A first-principles study. Comput. Mater. Sci. 2021, 188, 110131. [CrossRef]

57. Zhang, Y.; Feng, Y.; Zhu, B.; Zhao, J.; Jiang, T. Graphene based tunable metamaterial absorber and polarization modulation in terahertz frequency. Opt. Exp. 2014, 22, 22743-22752. [CrossRef] [PubMed]

58. Su, Z.; Yin, J.; Zhao, X. Terahertz dual-band metamaterial absorber based on graphene/MgF $\mathrm{M}_{2}$ multilayer structures. Opt. Exp. 2015, 23, 1679-1690. [CrossRef] [PubMed]

59. Yao, G.; Ling, F.; Yue, J.; Luo, C.; Ji, J.; Yao, J. Dual-band tunable perfect metamaterial absorber in the THz range. Opt. Express 2016, 24, 1518-1527. [CrossRef] [PubMed]

60. Li, H.G.; Ji, C.S.; Ren, Y.Z.; Hu, J.; Qin, M.; Wang, L. Investigation of multiband plasmonic metamaterial perfect absorbers based on graphene ribbons by the phase-coupled method. Carbon 2019, 141, 481-487. [CrossRef]

61. Yao, Y.; Liao, Z.; Liu, Z.; Liu, X.; Zhou, J.; Liu, G.; Yi, Z.; Wang, J. Recent progresses on metamaterials for optical absorption and sensing: A review. J. Phys. D Appl. Phys. 2021, 54, 113002. [CrossRef]

62. Huang, X.; Yang, F.; Gao, B.; Yang, Q.; Wu, J.; He, W. Metamaterial absorber with independently tunable amplitude and frequency in the terahertz regime. Opt. Express 2019, 27, 25902-25911. [CrossRef] [PubMed]

63. Ke, S.L.; Wang, B.; Huang, H.; Long, H.; Wang, K.; Lu, P.X. Plasmonic absorption enhancement in periodic cross-shaped graphene arrays. Opt. Express 2015, 23, 8888-8900. [CrossRef] [PubMed]

64. Rodrigo, D.; Limaj, O.; Janner, D.; Etezadi, D.; de Abajo, F.J.G.; Pruneri, V.; Altug, H. Mid-infrared plasmonic biosensing with graphene. Science 2015, 349, 165-168. [CrossRef] [PubMed]

65. Yu, J.; Zhu, J.; Ye, S.; Wang, X. Ultra-wide sensing range plasmonic refractive index sensor based on a two-dimensional circular-hole grating engraved on a gold film. Results Phys. 2021, 26, 104396. [CrossRef]

66. Xiang, Y.J.; Dai, X.Y.; Guo, J.; Zhang, H.; Wen, S.; Tang, D. Critical coupling with graphene-based hyperbolic metamaterials. Sci. Rep. 2014, 4, 5483. [CrossRef]

67. Christopoulos, T.; Tsilpakos, O.; Sinatkas, G.; Kriezis, E.E. On the calculation of the quality factor in contemporary photonic resonant structures. Opt. Express 2019, 360479. [CrossRef]

68. Shuang, W.; Sheng, L.J. Hollow-petal graphene metasurface for broadband tunable THz absorption. Appl. Opt. 2019, 58, 3023-3028. [CrossRef]

69. Wang, J.; Hu, C.P.; Tian, Q.; Yu, W.X.; Tian, H.H. Ultrahigh-Q and polarization-independent terahertz metamaterial perfect absorber. Plasmonics 2020, 15, 1943-1947. [CrossRef]

70. Wu, D.; Wang, M.; Feng, H.; Xu, Z.; Liu, Y.; Xia, F.; Zhang, K.; Kong, W.; Dong, L.; Yun, M. Independently tunable perfect absorber based on the plasmonic in double-layer graphene structure. Carbon 2019, 155, 618-623. [CrossRef]

71. Zhou, N.; Xu, X.F. Metamaterial-based perfect absorbers for efficiently enhancing near field radiative heat transfer. J. Quant. Spectrosc. Radiat. Transf. 2015, 167, 156-163. [CrossRef]

72. Zhang, J.; Zhu, Z.; Liu, W.; Yuan, X.; Qin, S. Towards photodetection with high efficiency and tunable spectral selectivity: Graphene plasmonics for light trapping and absorption engineering. Nanoscale 2015, 7, 13530-13536. [CrossRef] [PubMed] 